\title{
Using UWB Radios as Sensors for Disaster Recovery
}

\author{
Jeongeun Julie Lee and Suresh Singh \\ Department of Computer Science \\ Portland State University \\ Portland, OR 97207 \\ Email: jeong.julie.lee@intel.com,singh@cs.pdx.edu
}

\begin{abstract}
This paper considers the problem estimating the interior structure of a collapsed building by using embedded UWB radios as sensors. We created an extensive database of UWB propagation data through various building materials. Then, using this data and a novel algorithm, we demonstrate that we can indeed determine material type, thickness and cavity dimensions using UWB radios. This paper presents the algorithm and the evaluation results. As we show, for most common building materials such as concrete and reinforced concrete, the presented algorithm has a very small estimation error.
\end{abstract}

\section{INTRODUCTION}

Rescuing trapped survivors after a building collapses following an earthquake is a difficult and slow task. The primary reason for this is that rescuers do not know the structural stability of the rubble and thus need to excavate slowly and deliberately. For trapped survivors, this slow excavation can prove fatal. Tools to determine the location of survivors and the structural topology of the collapsed building typically consist of probes or robots lowered through holes. Unfortunately, these tools can only survey a piece of the whole structure and are typically restricted to mapping the top part of the collapse.

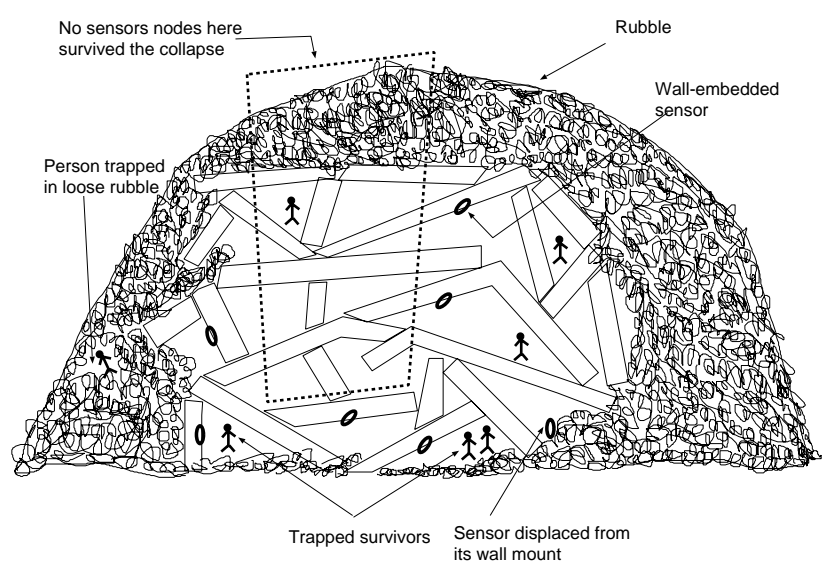

Fig. 1. Cutaway view of the building's interior.

In our research we are developing sensors that are embedded in the walls of the building during construction (or retrofitted later). These sensors consist of a UWB component for communication and sensing as well as an ultrasonic sensor and

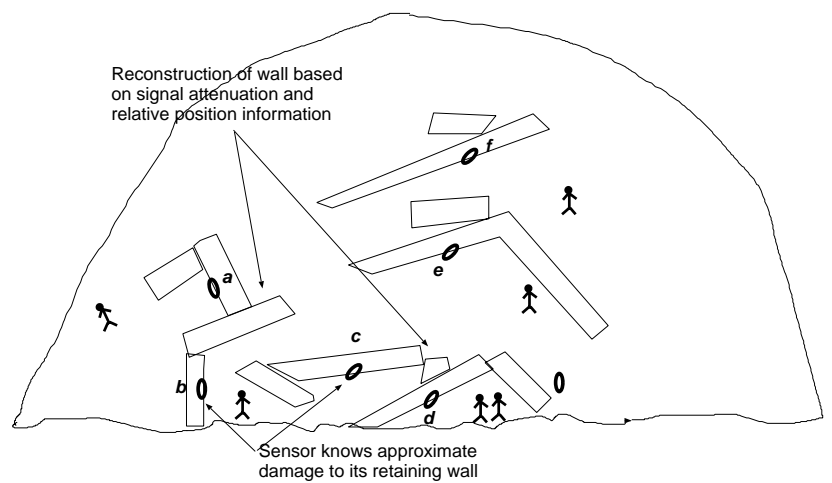

Fig. 2. Representation of the building's interior produced by our system.

heartbeat sensor. The idea is that when the building collapses, these sensors will collaboratively sense the interior of the collapsed structure and pass this information to the surface where software fuses the data to create a three dimensional view of the building's interior. Figure 1 shows a view of the interior of the collapsed building. As shown, there are several survivors located in different cavities (at different depths) as well as a complicated arrangement of walls that form the support for the whole structure. It is easy to see that by carelessly removing the wrong piece of the rubble, we may precipitate a secondary collapse with fatal consequences. Thus, the goal of our system is to develop as detailed a map of the interior as possible in order to give rescuers hints about how to excavate.

In a collapse, we expect many sensors to be destroyed. Furthermore, the location of the remaining sensors vis a vis the rubble, will limit the ability of our system to provide as detailed a map as illustrated in Figure 1. However, even a partial map such as Figure 2 that can be produced by our system will be very beneficial to rescuers. This map shows the location of various survivors as well as the primary support walls in the structure. It is easy to see that developing this form of a map requires us to solve several sensing problems including detection of people via heartbeats or $\mathrm{CO}_{2}$ levels, identification of cavity shapes, determination of the supporting 
wall composition (thickness) and orientation, etc. All this sensed information is then fused together to create a map like Figure 2.

In this paper, we address only one sensing sub-problem of this larger project. Specifically, we examine the problem of determining wall composition, thickness and location (with respect to sensors). For this purpose, we rely on the propagation properties of the UWB radios. The remainder of this paper is organized as follows. In the next section we survey related work on material sensing. Section III presents the approach we use for sensing and section IV describe the results of our experiments.

\section{RELATED WORK}

UWB transmitter localization effort using Time Difference of Arrival (TDOA) with multiple antenna pairs was conducted by Young, et al. [1], where the measurement was made in an anechoic chamber (W13xD20xH12m) placing trees or metallic objects in different positions of the anechoic chamber floor to create a multipath environment. The transceivers in this study had an LOS path, whereas signals in our study had non-line-ofsight (NLOS) harsher propagation conditions because of travel through building materials. Attempts to reduce the localization error in the NLOS case were made, e.g., [2], [3], [4], but excessive propagation delay in the NLOS condition was a limiting factor of precise ranging [3]. Wylie and Holtzman [2] used statistical information of the difference in the variance of time of arrival (TOA) in the line-of-sight (LOS) case and in the NLOS case. Algorithms that detects the first path under a multipath condition were developed, e.g., [3], [4]. Our algorithm estimates location of a transmitter with very small error even with the considerable ranging error caused by excessive propagation delay in the NLOS case overcoming ranging limitation.

Time Domain Corporation [5] received a patent on wideband radar technology that detects the presence of an object or motion through a wall, which can be used in through the wall sensing. Through-wall sensing of heart beat using Direction of Arrival (DOA) and reflection was previously proposed by Chia, et al. [9].

Technologies other than UWB such as X-ray tomographic imaging [8] and Ground Penetrating Radar (GPR) [6], [7] are being applied to determine concrete thickness, to create an image of the concrete interior structure, to detect fractures, and other applications. GPR gained its popularity over X-ray tomographic imaging due to its low cost operation and safeness. However, not much work has been done that determines wall thickness using UWB technology, which is critical in locating transceivers precisely and in creating the image of building or rubble structure.

\section{RESEARCH APPROACH}

Figure 3 illustrates the problem being considered in this paper. Assume there is a cavity that contains one sensor. In order to create a map as in Figure 2, we need to determine the size and shape of the cavity as well as the composition and

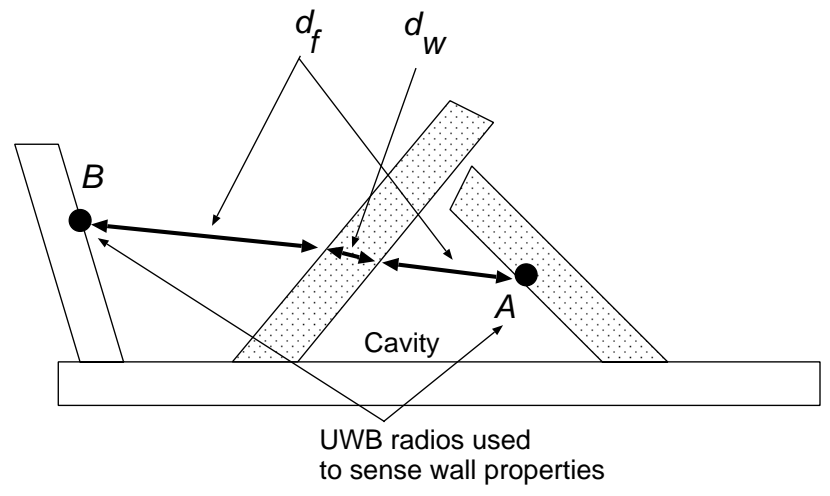

Fig. 3. Determining wall thickness of a cavity.

thickness of each of the walls forming the cavity ([10] uses a moving transmitter and fixed receiver to produce a propagation map of a room). The problem of mapping the interior of the cavity is done by a ultrasonic sensor and is out of scope of this paper. The problem we consider here is determining the thickness and type of each wall.

Given a sufficiently dense deployment of sensors in the building, there is a good chance that another sensor will be present outside the cavity (sensor $B$ as shown in the figure). We now use the UWB radio to determine the composition and thickness of the shaded wall as follows:

1) Let us assume that the wall thickness is $d_{w}$ and the sum of the two distances between each radio and the wall is $d_{f}$ (freespace distance). Thus, the distance between the two radios $A$ and $B$ is $y=d_{f}+d_{w}$ (see Figure 3 ).

2) Sensor $B$ transmits a signal at a pre-determined power. We assume that sensor $A$ either has two receivers or two antennas separated by some distance. The two values of the received power are $p_{1}$ and $p_{2}$. Let the time difference of when each antenna receives the signal be $\delta t$ and we set $x=c \delta t \mathrm{~m}-x$ indicates the difference in distances of the two antennas or receivers from the transmitter. $c$ is the speed of light in freespace. Let us arbitrarily assume that the signal is received at antenna 1 first (i.e., it is closer to $B$ ).

3) Generally the material used for construction is known apriori. Indeed, the main load-bearing members used typically have well-defined thicknesses and composition (e.g., 8 inch concrete etc.). Assume that we characterize the path loss seen between two radios separated by some distance $D$ and with one piece of building material (of various thicknesses) in between. We can plot the received signal strength (for a given transmit power) versus $D$ for different materials as shown in Figure 4.

4) The distance $y_{1}$ (i.e., distance to closer receiver) is an unknown. However, we know that at distance $y_{1}$ the received power is $p_{1}$ and at $y_{2}=y_{1}+x$ it is $p_{2}$. We draw a horizontal line segment in Figure 4 corresponding to power levels $p_{1}$ and $p_{2}$. We guess a value for $y_{1}$ (call it $\bar{y}_{1}$ ) and construct a line segment connecting points 
$\left(\bar{y}_{1}, p_{1}\right)$ and $\left(\bar{y}_{2}, p_{2}\right)$. We compute the Mean Square Error (MSE) between this line segment and the path loss line for each material. The range of values for $\bar{y}_{1}$ goes from zero to $y_{\max }$ where $y_{\max }$ is determined using time of arrival data from the UWB radio. Assuming freespace, the ToA information gives us an upper bound on $\bar{y}_{1}$ (if material is present then $\bar{y}_{1}$ is smaller since speed of the signal is less in material) and this is the value used for $y_{\max }$.

5) Among all the potential fits, we select the one that minimizes the MSE. This gives us a good estimate for $y_{1}$ as well the material and the thickness (read off from the path loss curves).

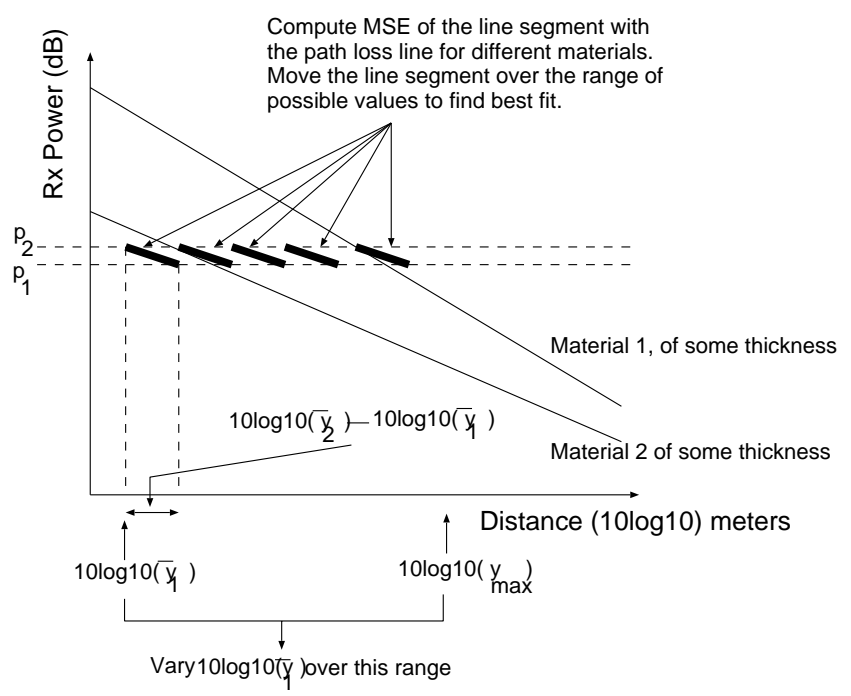

Fig. 4. Illustration of the algorithm.

\section{EXPERIMENTAL RESULTS}



Fig. 5. Laboratory setup.

In order to verify that our algorithm to determine the composition and thickness of material works, we conducted a series of very detailed signal propagation measurements. There are two main steps in our experimental work.

1) Characterize the pathloss for a variety of building material in order to produce a graph like Figure 4.

2) Run experiments to identify the type and thickness of the wall as described in the algorithm.

In order to characterize the pathloss we used the experimental setup shown in Figure 5. We use PulseOn 210 UWB radios from Time Domain Corporation. The transmitter is shown sitting on a stool in the figure while the receiver is in the enclosed box that has shielding material layered on the inside (90dB attenuation). The building material is mounted between the two radios and the transmitter was put at different distances. The received power was recorded (ten repititions at each distance) for each placement. For our measurements we used standard types of building material including:

- Concrete - 4.5, 6.5, 8 inches thick

- Reinforced concrete - 7.5 inches

- Drywall - 0.5 inches

- Plywood - 0.5 inches

In addition we considered combinations of material:

- Freespace

- Concrete \& drywall

- Concrete \& plywood

- Concrete \& plywood \& drywall

- Reinforced Concrete \& drywall

- Reinforced Concrete \& plywood

- Reinforced Concrete \& plywood \& drywall

- A metallic grid \& concrete

Finally, we conducted measurements when the material was dry as well as wet.

The measurements we conducted were aimed at being as representative of what we may find in the field (combinations of material as well as wet conditions). Furthermore, we deliberately used the placement of the radios and material as in Figure 5 since in a cavity, Figure 3, the two radios will have freespace between themselves and the wall and there will be all kinds of multipath present. The data we collected is available at http://rescuenet.cs.pdx.edu for general use (the measurements in the database used the Time Domain P200 radios). In this paper we do not provide an analysis of this extensive database but rather focus on the use of the database for the purpose of identifying material properties.

\section{A. Determining material thickness and type}

Figure 6 plots the pathloss for freespace, reinforced concrete (7.5in), concrete (6.5in) and wood (4.5in). The numbers next to each line indicate the slope and the y-intercept. For clarity we have left out the individual measurements and only retained the best fit lines.

In order to test out algorithm, we used the following experimental methodology for each material:

- We use two receivers and one transmitter. Let $y_{1}$ and $y_{2}$ denote the distance of the two receivers from the transmitter. 


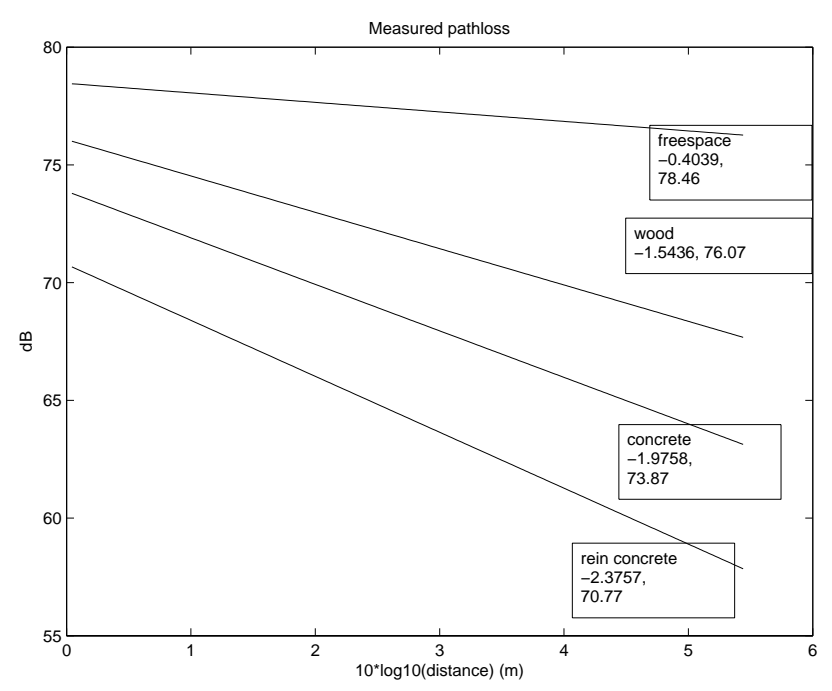

Fig. 6. Pathloss for a subset of materials.

- We vary $y_{1}$ between 1 and 3 meters (to better represent small cavities found in fallen buildings). $y_{2}$ also varies between these values but is greater than $y_{1}$. In all there were 450 pairwise measurement points. For each $y_{1}, y_{2}$ value, we collected 5 measurements of received power $p$ and ToA per receiver. Thus, we collected a total of $450 \times 5 \times 5$ data points.

- For each pair of $p_{1}, p_{2}$ values, we run the algorithm described in section III and find:

- Type of material and thickness (the answer is a 1 or 0 depending on whether we guess correctly or incorrectly).

- The estimated value of $y_{1}$ (since this is also unknown in reality).

Table I summarizes the main results for three materials. As we can see, the algorithm correctly determines the material type and thickness almost all the time. Wood was the only material where the algorithm made some mistakes. This is due to the fact that wood does not attenuate the signal much as compared to concrete or reinforced concrete and thus the algorithm sometimes concludes freespace as the material rather than wood. The table also summarizes the error in estimating $y_{1}$. Notably, wood has the highest error while for reinforced concrete the error is negligible. Indeed, in the case of wood, the algorithm always underestimates the value of $y_{1}$.

In order to understand the error in distance estimate, we plot the error (expressed as a percentage) in estimating $y_{1}$ versus $y_{2}-y_{1}$ (i.e, the distance between the two receivers). Figure 7 shows the plot for freespace and Figure 8 shows the same plot for reinforced concrete. The error for freespace is highly variable for all $y_{1}-y_{2}$ values and is explained by the severe multipath present in the laboratory which is essentially a large concrete sub-basement used for testing concrete structures. Interestingly, the error is small and consistent for reinforced concrete primarily because a great deal of signal energy is absorbed by the reinforced concrete leaving little energy in various reflected components. In view of our original application, this is good news since we can conclude that in the field our algorithm ought to provide good estimates.

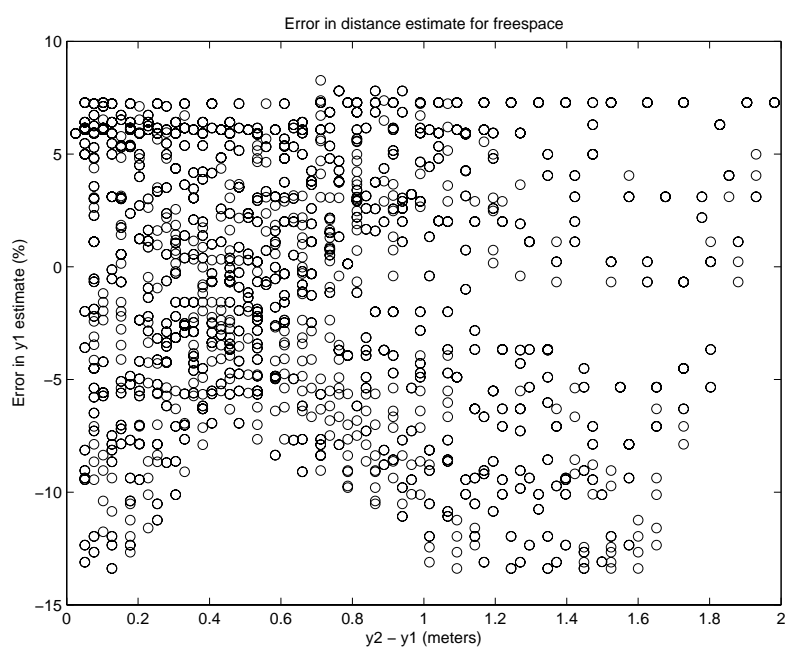

Fig. 7. Error in distance estimate for freespace.

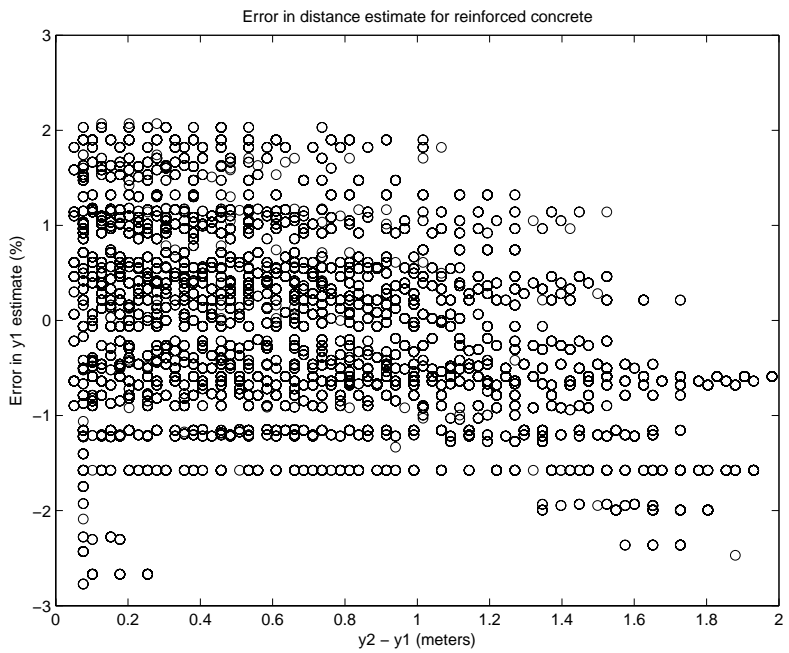

Fig. 8. Error in distance estimate for reinforced concrete.

\section{CONCLUSIONS}

In this paper we consider the problem of estimating the thickness and type of material using UWB radios as sensors. The algorithm developed is shown to have very good performance and it is able to identify the material correctly almost always. The approach used in this paper utilized two radio receivers separated by some distance. As a next step we will consider whether the accuracy can be further improved by utilizing the ultrasonic ranging data that is available to us.

\section{REFERENCES}

[1] D. P. Young, C. M. Keller, D. W. Bliss, and K. W. Forsythe, "UltraWideband (UWB) Transmitter Location Using Time Difference of Arrival (TDOA) Techniques," Proceedings of the Thirty-Seventh Asilomar Conference on Signals, Systems, and Computers, Pacific Grove, CA, Nov. 9-12, 2003, pp. 1225-1229. 


\begin{tabular}{|l|c|c|}
\hline & Material thickness \& type guess & Mean absolute Error in $y_{1}$ estimate \\
\hline Freespace & $100 \%$ & $4.84 \%$ \\
\hline Concrete & $99.39 \%$ & $3.39 \%$ \\
\hline $\begin{array}{l}\text { Reinforced } \\
\text { Concrete }\end{array}$ & $99.95 \%$ & $0.76 \%$ \\
\hline Wood & $98.7 \%$ & $15.7 \%$ \\
\hline
\end{tabular}

TABLE I

SUMMARY OF ALGORITHM PERFORMANCE.

[2] M.P. Wylie and J. Holtzman, "The non-line of sight problem in mobile location estimation", Proc. 5th IEEE Int. Conf. Universal Personal Communications, Cambridge, MA, Sept. 1996, vol. 2, pp. 827-831.

[3] JY. Lee and R.A. Scholtz, "Ranging in a dense multipath environment using an UWB radio link", IEEE Journal Select. Areas Commun., Dec. 2002, vol. 20, no. 9, pp.1677-1683.

[4] B. Dennis, J. Keignart, and N. Daniele, "Impact of NLOS propagation upon ranging precision in UWB systems", Proc. IEEE Conf. Ultra Wideband Systems and Technologies (UWBST'03), Reston, VA, Nov. 2003, pp. 379-383.

[5] Time Domain Corporation, "Time domain radio transmission system", US Patent No. 7,030,806, Apr. 2006, "www.uspto.gov".

[6] J. E. Mast and E. M. Johansson, "Three-dimensional ground penetrating radar imaging using multi-frequency diffraction tomography”, SPIE, vol. 2275, Jul. 1994

[7] J. Mat, "Nondestructive Evaluation of Concrete Infrastructure with Ground Penetrating Radar", Civ. Engineering, vol. 15, issue 3, May/Jun. 2003 , pp. 287-297.

[8] E.J. Garboczi, "Three-dimensional mathematical analysis of particle shape using X-ray tomography and spherical harmonics: application to aggregates used in concrete", Cern. Concr. Res., 32, 2002, pp. 16211638.

[9] M.Y.W. Chia, S.W. Leong, C.K. Sim, K.M. Chan, "Through-wall UWB radar operating within FCC's mask for sensing heart beat and breathing rate", European Radar Conference, Oct. 2005, pp 267-270.

[10] R. Zetik, J. Sachs and R. Thoma, "Imaging of propagation environment by UWB channel sounding", COST273 Temporary Document TD(05) 058, Bologna, Italy, January 2005. 